\title{
Unitarity of neutrino mixing matrix
}

\author{
Ha Nguyen Thi Kim ${ }^{1,2}$, Van Nguyen Thi Hong ${ }^{2,3}$, and Son Cao Van ${ }^{4}$ \\ ${ }^{1}$ Ho Chi Minh University of Science, Ho Chi Minh City, Viet Nam \\ ${ }^{2}$ Institute of Physics (IOP), Ha Noi, Viet Nam \\ ${ }^{3}$ Institute For Interdisciplinary Research in Science and Education (ICISE), Quy Nhon, Viet Nam \\ ${ }^{4}$ High Energy Accelerator Research Organization (KEK), Tsukuba, Ibaraki, Japan
}

\begin{abstract}
Neutrinos are neutral leptons and there exist three types of neutrinos (electron neutrinos $v_{e}$, muon neutrinos $v_{\mu}$ and tau neutrinos $v_{\tau}$ ). These classifications are referred to as neutrinos's "flavors". Oscillations between the different flavors are known as neutrino oscillations, which occurs when neutrinos have mass and non-zero mixing. Neutrino mixing is governed by the PMNS mixing matrix. The PMNS mixing matrix is constructed as the product of three independent rotations. With that, we can describe the numerical parameters of the matrix in a graphical form called the unitary triangle, giving rise to $\mathrm{CP}$ violation. We can calculate the four parameters of the mixing matrix to draw the unitary triangle. The area of the triangle is a measure of the amount of $\mathrm{CP}$ violation.
\end{abstract}

\section{Neutrino Oscillation}

Neutrino oscillations are a results of neutrino mixing via: the left-handed flavor neutrino fields. Neutrino flavor eigenstates can be expressed as a superposition of the mass eigenstates

$$
v_{\alpha L}=\sum_{i=1}^{n} U_{\alpha i} v_{i L},
$$

where

$v_{i}(i=1,2,3)$ refers to the mass eigenstates, $v_{\alpha}(\alpha=e, \mu, \tau)$ refers to the flavor eigenstates.

$U$ is the Pontecorvo-Maki-Nakagawa-Sakata matrix, where each element is a complex number

$$
\begin{aligned}
U_{P M N S} & =\left(\begin{array}{ccc}
1 & 0 & 0 \\
0 & c_{23} & s_{23} \\
0 & -s_{23} & c_{23}
\end{array}\right)\left(\begin{array}{ccc}
c_{13} & 0 & s_{13} e^{-i \delta} \\
0 & 1 & 0 \\
-s_{13} e^{i \delta} & 0 & c_{13}
\end{array}\right)\left(\begin{array}{ccc}
c_{12} & s_{2} & 0 \\
-s_{12} & c_{12} & 0 \\
0 & 0 & 1
\end{array}\right) P \\
& =\left(\begin{array}{ccc}
c_{12} c_{13} & s_{12} c_{13} & s_{13} e^{-i \delta_{C P}} \\
-s_{12} c_{23}-c_{12} s_{23} s_{13} e^{i \delta_{C P}} & c_{12} c_{23}-s_{12} s_{23} s_{13} e^{i \delta_{C P}} & s_{23} c_{13} \\
s_{12} s_{23}-c_{12} c_{23} s_{13} e^{i \delta_{C P}} & -c_{12} s_{23}-s_{12} c_{23} s_{13} e^{i \delta_{C P}} & c_{23} c_{13}
\end{array}\right) P
\end{aligned}
$$

where

$\theta_{i j}$ is the mixing angle, $c_{i j}=\cos \theta_{i j}$ and $s_{i j}=\sin \theta_{i j}$,

CP-violating phases: Dirac phase $\delta_{C P}$ has $P=1$ and Majorana phases $\phi_{1}, \phi_{2}$ has $P=$ $\operatorname{diag}\left(1, e^{i \frac{\phi_{1}}{2}}, e^{i \frac{\phi_{2}}{2}}\right)$ 


\section{Oscillation probability}

The oscillation probability of flavor transition $v_{\alpha} \rightarrow v_{\beta}$ after distance $L$ in vacuum and with energy $E$

$$
\begin{array}{r}
P_{\dot{v}_{\alpha} \rightarrow v_{\beta}}=\left|A_{\substack{(-) \\
v_{\alpha} \rightarrow v_{\beta}}}\right|^{2}=\delta_{\alpha \beta}-4 \sum_{j>i} \operatorname{Re}\left(U_{\alpha i}^{*} U_{\beta i} U_{\alpha j} U_{\beta j}^{*}\right) \sin ^{2}\left(\frac{\Delta m_{i j}^{2} L}{4 E}\right) \\
\mp 2 \sum_{j>i} \operatorname{Im}\left(U_{\alpha i}^{*} U_{\beta i} U_{\alpha j} U_{\beta j}^{*}\right) \sin \left(\frac{\Delta m_{i j}^{2} L}{2 E}\right),
\end{array}
$$

where $\Delta m_{i j}^{2}=m_{i}^{2}-m_{j}^{2}$,

- $m_{1}<m_{2}<m_{3}$ corresponds to a normal mass order (NO),

$-m_{3}<m_{1}<m_{2}$ corresponds to a inverted mass order (IO).

\section{Unitarity triangle}

The PMNS matrix is unitary and so $U^{\dagger} U=U U^{\dagger}=1$. From this property, we can normalize the matrix elements such that $\sum U_{\alpha i} U_{\alpha i}^{*}=1$. So, there are 6 triangles governed by the orthogonal relations

$$
\begin{array}{ll}
\sum U_{\alpha i} U_{\beta i}^{*}=0, & (\alpha \neq \beta) \\
\sum U_{\alpha i} U_{\alpha j}^{*}=0 . & (i \neq j)
\end{array}
$$

\section{Results}

\subsection{The unitary triangle with our current neutrino landscape}

The current best-fit values from NuFit.org [3] are given in following table. The numbers in the second and third column are assuming Normal Ordering or Inverted Ordering respectively and the numbers given are relative to the respective global minimums.

Table 4.1: The current best fit values from NuFit.org (Global fit framework for $v$ oscillation) on November 2017.

\begin{tabular}{ccc}
\hline & Normal Ordering (NO) & Inverted Ordering (IO) \\
\hline & best fit $\pm 1 \sigma$ & best fit $\pm 1 \sigma$ \\
\hline $\sin ^{2} \theta_{12}$ & $0.307_{-0.012}^{+0.013}$ & $0.307_{-0.012}^{+0.013}$ \\
\hline $\sin ^{2} \theta_{23}$ & $0.538_{-0.069}^{+0.038}$ & $0.554_{-0.033}^{+0.023}$ \\
\hline $\sin ^{2} \theta_{13}$ & $0.02206_{-0.00075}^{+0.00075}$ & $0.02227_{-0.00074}^{+0.00074}$ \\
\hline$\delta_{C P}{ }^{o}$ & $234_{-31}^{+43}$ & $278_{-29}^{+26}$ \\
\hline
\end{tabular}


The values of PMNS matrix parameters are calculated as

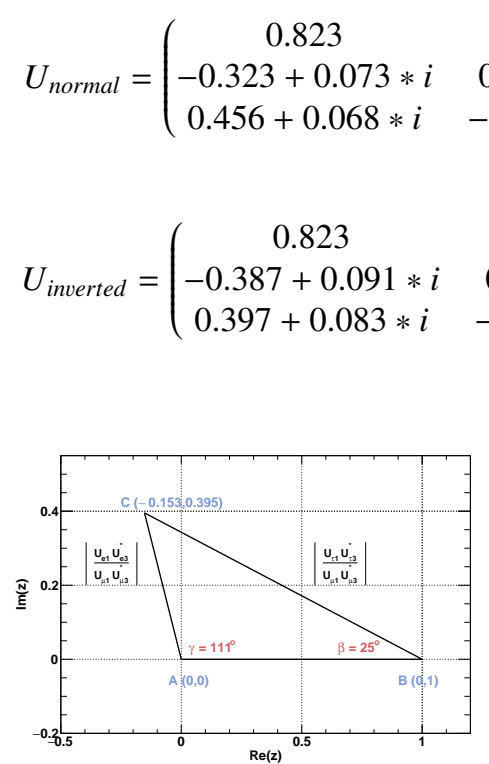

(a) NO

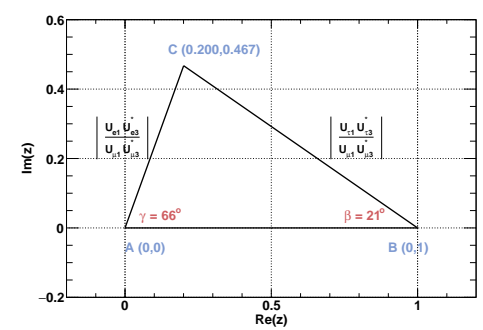

(b) IO

Figure 4.1: The unitary triangle for normal hierarchy and for inverted hierarchy corresponds to the unitary conditions on the first and third columns

\subsection{Simple model for estimating uncertainty}

These are the $1 \sigma$ ranges for the magnitudes of the matrix parameters, and the two values correspond to the global minimum and maximum values respectively.

$$
|U|=\left(\begin{array}{lll}
0.815 \rightarrow 0.831 & 0.537 \rightarrow 0.559 & 0.146 \rightarrow 0.151 \\
0.320 \rightarrow 0.393 & 0.536 \rightarrow 0.662 & 0.678 \rightarrow 0.747 \\
0.425 \rightarrow 0.456 & 0.552 \rightarrow 0.632 & 0.647 \rightarrow 0.721
\end{array}\right)
$$

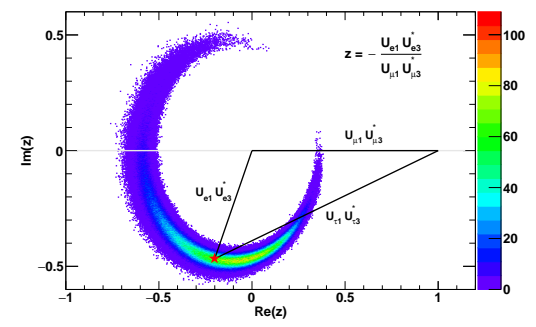

(a) $\mathrm{NO}$

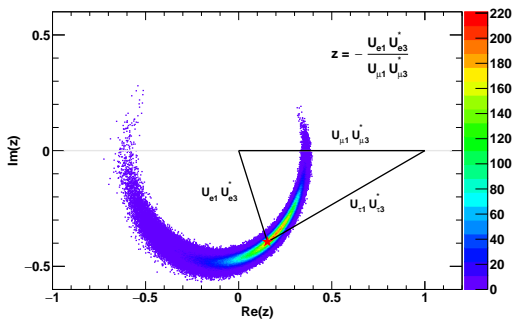

(b) IO

Figure 4.2: Plot of $\delta_{C P}$ with the four oscillation parameters having $1 \sigma$ uncertainties 


\subsection{Unitarity test with future neutrino experiments}
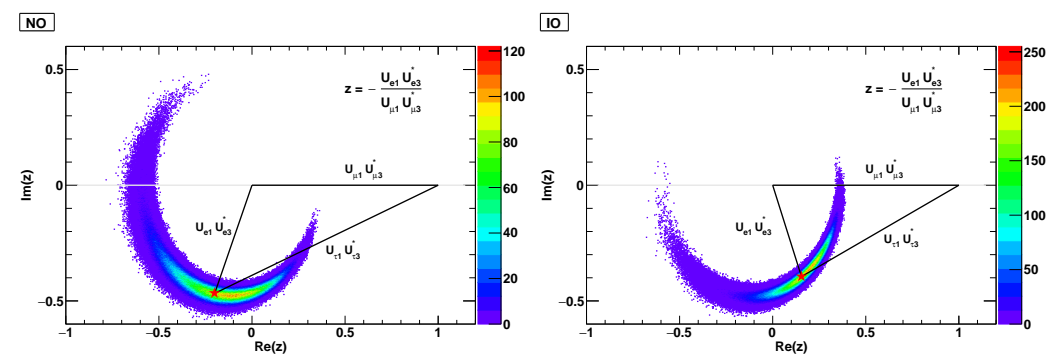

Figure 4.3: Plot of $\delta_{C P}$ with the four oscillation parameters having $1 \sigma$ uncertainties of $\pm 23^{\circ}$. (Hyper-K)
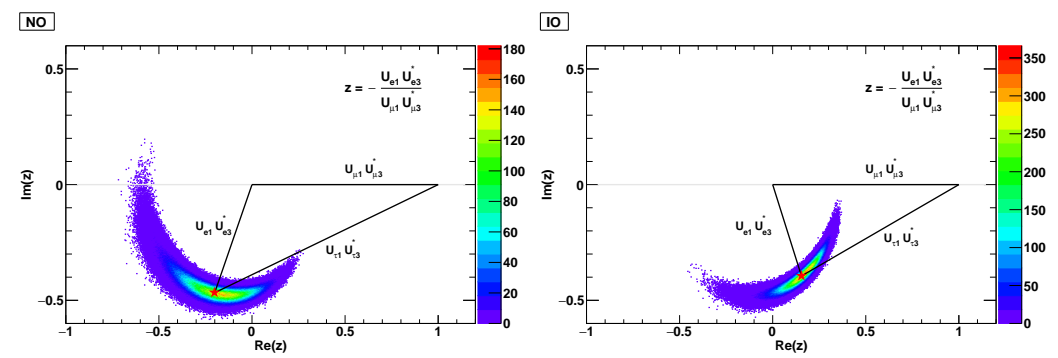

Figure 4.4: Plot of $\delta_{C P}$ with the four oscillation parameters having with $1 \sigma$ uncertainties of $\pm 15^{\circ}$. (Combining Hyper-K and DUNE)

\section{Conclusion}

- These six unitary triangles have the same area which is equal to half of Jarlskog's invariant, showing the $\mathrm{CP}$ violation of the process.

- By reducing uncertainties of $\delta_{C P}$, the size of the allowed region at the $\mathrm{C}$ vertex of the unitary triangle at some confidence level will be reduced. The precision of the unitary vertex position will be increased.

\section{References}

[1] P. Lipari, 1st CERN - CLAF School of High-energy Physics, Itacuruca, Brazil (CERN2003-003), 115-200 (2001)

[2] Z. Xing and J. Zhu, Nucl. Phys. B 908, 302-317 (2016)

[3] NuFit, http://wwww.nu-fit.org

[4] Particle Data Group, http://pdg.lbl.gov 